\title{
Analysis on the Application Thought of 3DMAX in Animation Production
}

\author{
Walisi Abulizi, Hong Xu \\ Xinjiang University, Xinjiang 830000, China
}

\begin{abstract}
DMAX, a three-dimensional animation rendering and production software based on PC system, is produced by Discreet. Its existence has made the special effect of television and animation have revolutionary progress. Besides, it is widely used in the advertisement, television, industrial design, construction design, three-dimensional animation production and multi-media production, and it has the prospect of intelligent development. Based on the specific scenes of animation production, this paper analyzes the practical application of 3DMAX, and it refers to the application methods in the animation design, aiming at having better help for the application of 3DMAX and strengthening the practical application of 3DMAX in the field of animation design.
\end{abstract}

Keywords: 3DMAX; animation production; application thoughts.

\section{Introduction}

Along with the constant development of computer hardware technology and animation production software technology, the content and technology system of animation design tend to be perfect. And animation design refers to various contents, for example, story content, animation roles and scene design, which realizes the good presentation for the animation theme though the integration of contents from various aspects. At the same time, the development and expansion of animation industry are closely related with social culture and economy development. Besides, animation, as an emerging design industry, is known for the development in the design field. And the market demand of animation design works with high-quality is more obvious along with the constant promotion of production level and audience' appreciation abilities. While the key point deciding the excellent animation work is from the modern realization method. As an advanced tool, 3DMAX is convenient for the supplement and enhancement of scenes and special effects in the animation production, especially, its "standard" modeling process, which is of great significant to construct threedimensional animation characters and scenes, so this research has some practical values.

\section{Current Development Situation of Computer Graphics Technology}

With the constant development of network communication technology, computer animation production technology is becoming more and more developed, and the production of 3DMAX is of great significance to the improvement of animation production level. Firstly, its static drawing function uses various picture design tools, and it can quickly achieve the smoke effect, halo effect of burning candles, burning dust effect and smoke effect of cigarettes with its over 30 kinds of brushes, which is vital for those creative advertisements needing to express the dim or dark and high contrast degree. Apart form the creativity on the effect of graphics and pictures, 3DMAX can also recreate with the existing pictures and make the second handling with the methods of cutting, moving, recovering, changing, vignette and blurring. It is convenient for the designers to better achieve their own design concept on the basis of existing pictures and reduce the effect of the difficulty in handling with the existing materials on designers' work. Secondly, the accuracy of mobile beacon drawing technology is higher than static drawing, which draws mainly through the move of beacon nodes. The last one is the application of 3DMAX. It is mainly forecasting the sample information and constantly update the sample space and form one kind of distribution. Using the estimated node with the center to draw will not augment the error due to the moving of the node and also enhance the accuracy of pictures as well as reduce the cost and requirement with the hardware. 


\section{Application Thought Analysis of 3DMAX in the Animation Production}

\subsection{Apply 3DMAX to Express the Animation Quality}

The application of 3DMAX in the animation production can express the animation quality and create the sense of occasion and realize the specific theme. The detailed discussion is as follows:

\subsubsection{Express the Halo Effect of Animation and Strengthen the Sense of Occasion}

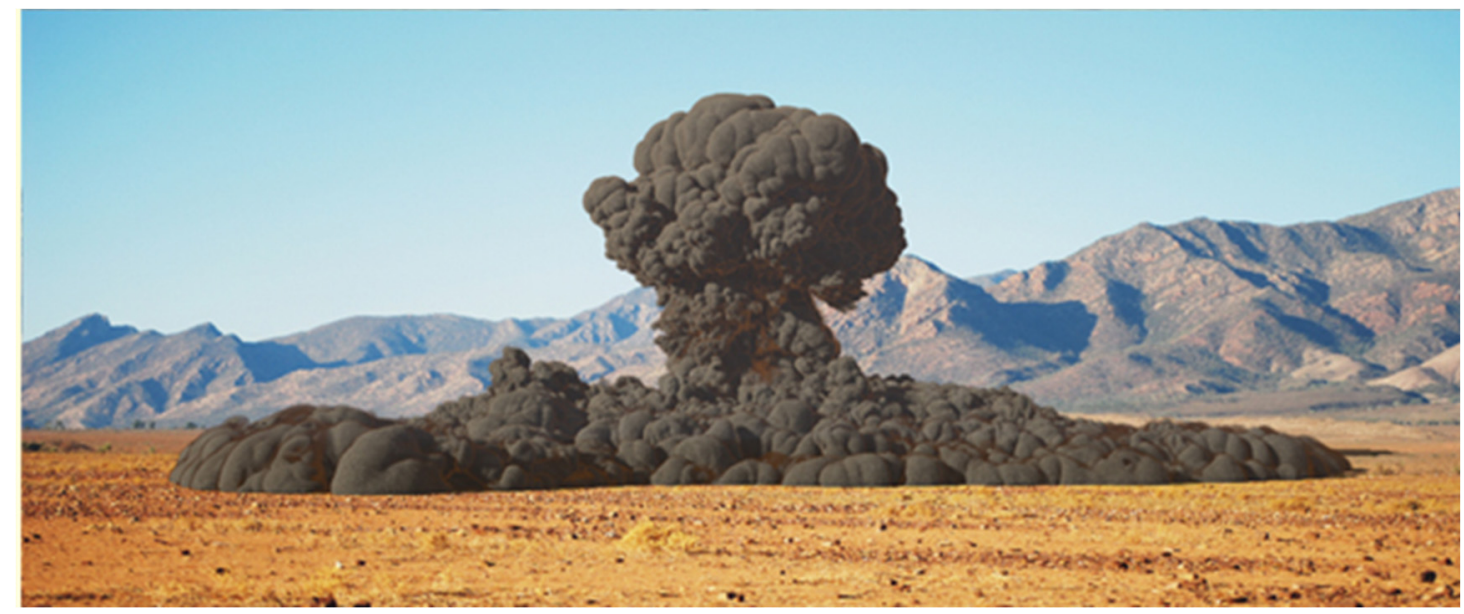

Figure 1. Mushroom cloud produced by $3 \mathrm{DMAX}+\mathrm{AE}$

The application of 3DMAX in the animation production can produce the halo effect with AE to strengthen the sense of occasion, for example, the mushroom cloud produced by the bomb exploding simulated in the above figure can best express the corresponding real scene. The combination with such special scene in the production of animation special effect production can better show the real meaning of "actually being there".

\subsubsection{Use 3DMAX to Match the Special Animation Theme}

Animation works are produced surrounding the special themes, and animation themes are the main connotation that the producers of animation works want to express and the entrance of the audience to understand the whole animation work. Distinct theme cannot be without certain presentation methods. In the former ones, the themes are mostly achieved by means of specific characters or things. However, the application of 3DMAX can achieve more unfettered themes, for instance, Avatar and Strategic Defense Initiative, they produced lots of space models through 3DMAX and realize the construction of virtual three-dimensional space through the simulation. They usually make the modeling of complex scene animation based on polygon.

\subsection{DMAX Shadow Art}

Visual elements mean the fundamental units to construct the visual objects and the tools and media for human being to receive and deliver information. Visual elements of plane design mainly include the picture, character and shape. Point, line, surface, color and space are the main presentation forms in the animation works. Visual elements can also be divided into the concrete ones and abstract ones. The particular visual element former by 3DMAX with the halo effect also has a good application, for example, the application of different colors. In fact, colors have the presentation effects of emotion and atmosphere, for instance, pink represents the quiet and romance, while gold is more likely to represent the elegance and brilliance. As the application of visual element, color has strong presentation. The visual feelings of people on the color will directly show on the plane design work. Color has the ideal effect on catching people's eyes. When people are looking at a plane design work, they will always be absorbed by its color brightness, abundance of matching and the fitness of mood at that moment, for example, people will firstly feel the visual pleasure and stimulate the sense experience. But large-scale contrast color and matching or conflict of cold and warm hues will have a good effect on the presentation of themes, which is same with the requirement of modern art. The 
presentation of rich color, high quality and high color saturation will make the modern plane design more easy to show designers' differentiated appealing and creativity, also providing more space for the latter production of graphics. It makes the style of the same works more diversified, and the presentation style, quality or mood more plural. An obvious contrast is the presentation degree of black-white scene and colorful scene, also showing the similar creativity design in the process of suing the color elements (as follows).

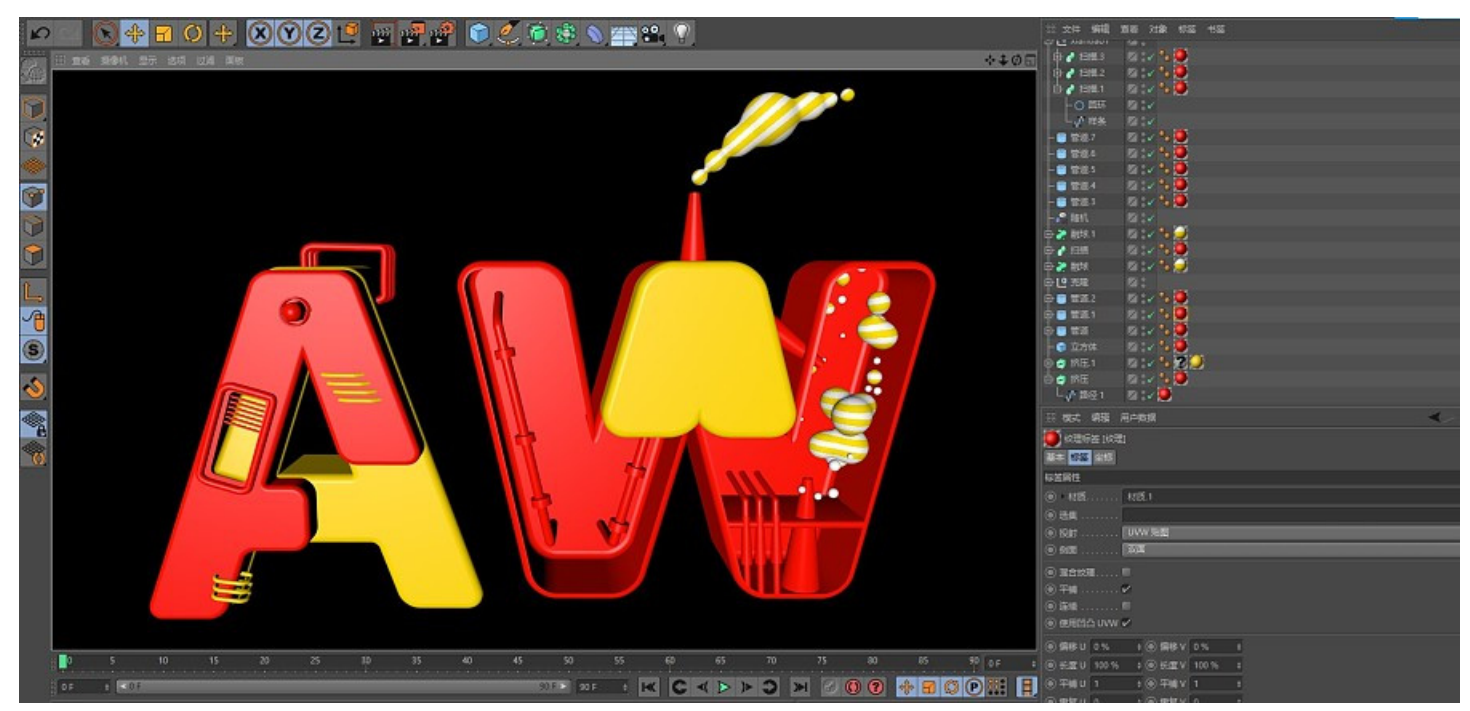

Figure 2. An Example of the Effect of Color Elements in Creative Design

\subsection{DMAX Dynamics}

Dynamics is one core function of 3DMAX, and designers can simulate the real and natural movement of objects through the dynamic model and computing method, not personally adjusting the dynamic scenes with complex structures. It can make the dynamic special effect in the animation become more vivid and abundant by systematically applying the dynamic special effect models such as the particle, cloth, hair and break, which will make the animation package elements more abundant to a great extent. Besides, 3DMAX dynamic effect can achieve the one that never been expressed, for example, the operation of enormous spacecraft, gear rotation of big machine, launching of rocket and flowing of lava. For example, the repeated movement of combustion cylinder piston expressed in the following figure. The dynamic picture achieved by the dynamic effect of 3DMAX can make the details of operated parts more abundant and diversified and better express the characteristics of animation works.

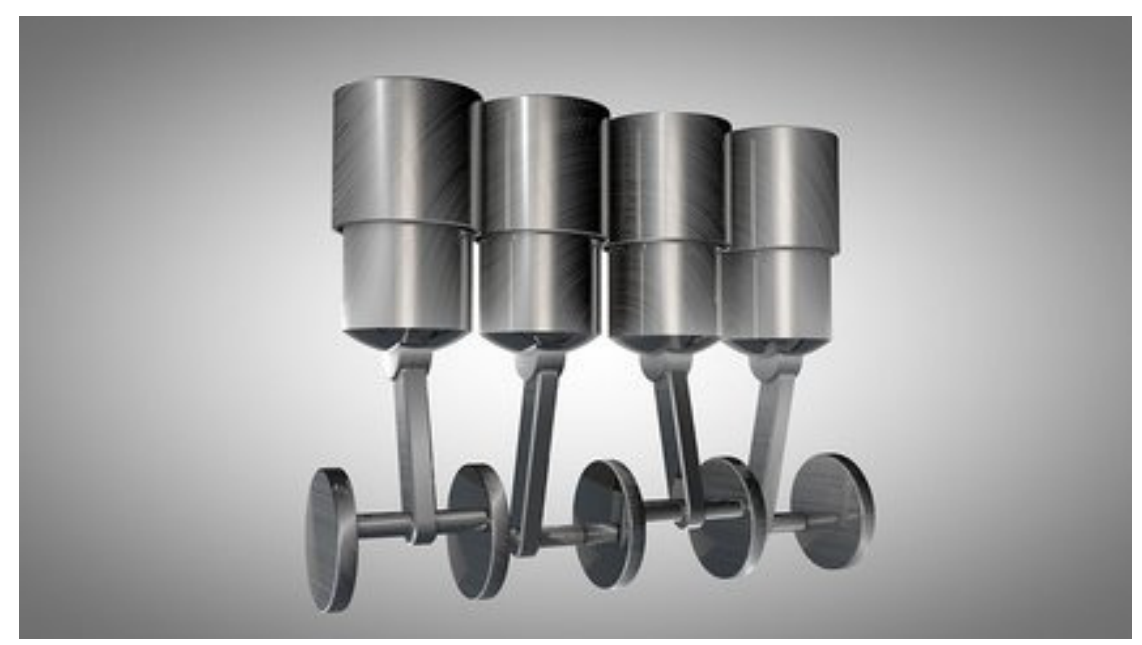

Figure 3. Reciprocating Motion Diagram of Cylinder Piston of Internal Combustion Engine 


\subsection{DMAX is used to Animation Packaging}

3DMAX three-dimensional animation simulates the movement method of real objects. It is used in the animation package to simulate the movement method of real objects, which can bring the vivid effect, and its accurate, real and limitless operation can better provide the new animation creativity presentation. For instance, the presentation with car movement, the traditional behavior is real shooting, however, which cannot achieve the creating effect of suffocating plots. But applying 3DMAX to handle with the key frame in the animation can realize the control with movement rhythm and create the presentation method of integrating fast and low. An obvious presentation is those animation works of wars will make the audience clearly see the swirling of bomb in its flying through the low flying scenes of bomb so that they can better understand the tense sense created by the shoot.

\section{Application Steps of 3DMAX}

\subsection{Design Related Modeling Photo Library}

Related graphics library is the main resource library in the application of 3DMAX, and the graphics and files allocation or the modeling construction will usually choose the related graphics library to finish. The reason is that its graphics are equipped with lots of advantages, for instance, convenient to program extension and deal with various pictures with large quantities and fast pace and easily expand or index. Graphics library can make the graphics connected with each other, greatly reduce the real work and compress the time of graphics modeling to make animation files greatly correspond to the characteristic of television production and have rather high resource intensity, which is of great significance to the animation development.

\subsection{Complete the Modeling with 3DMAX}

The modeling of 3DMAX needs to proceed with the graphics files provided by the manufacturers, and it has lots of information. While in the practical modeling process, these information in the whole graphics library won't be used at the same time, which will not only affect the normal operation of graphics library but be bad for the simplification of the structure. Therefore, it only needs to remain certain halo effects and information in the modeling and simplify the other parts so that the adjustment of graphics will be more easy and smooth. Memory function of 3DMAX graphics library will make the marks with this part of content and distinguish the modeling halo and make the use with dynamic graphics.

\subsection{Establish Related Resources Management Models}

Systematic management with animation in 3DMAX mainly proceeds with the method of resource management models. The static graphics, videos and characters in the animation will be managed in the corresponding resource management models. The specific operation methods include the inserting, amending and deleting. Character information management model mainly includes the place name and manage the character information in the system. Graphics information management model is close with character, mainly used to present the knowledge that includes the name of graphics and related knowledge information. Streaming media mainly transmits the continuous recording and video information to the network server after the handling to make it easy for the user to download and watch.

In the real operation of three-dimensional objects, 3DMAX is usually the most valuable, and it is not only much easier but making the whole presentation effect more vivid, when compared with traditional animation production process. Designers can set the specific achievement methods of different animation bodies through operating 3DMAX, for instance, the animation design of The Monkey King used the software to simulate the real construction scene design style to make the overall presentation similar with the real one, including the usage of style, pattern and materials. In order to greatly protrude the real sense of construction, the real one should be regarded as the blueprint to design. Such kind of scene design style includes rather heavy regional style and era characteristics, 
able to form the good visual guidance for the audience and clearly understand the real age and region. It has some intuition. At the same time, in order to create better atmosphere, 3DMAX also pays attention to the use of light to render the scene and form a complete $3 \mathrm{D}$ animation figure through the intention and reconstruction with the graphics of many models to make the presentation effect of the whole picture more vivid. After the establishment of model graphics library, animation model and last rendering, the basic animation elements can be presented, and then the dynamic tools of 3DMAX can be used to make the static graphics element acquire the similar dynamic impressions with the real moving objects and enhance the overall quality of animation.

\section{Conclusion and Prospect}

In conclusion, people's mental awareness level is constantly enhancing along with the social development and progress. The arrival of the reading era has brought the animation design a new development space and concept, and people are more looking forward to the colorful animation scenes and abundant themes. Animation is not only an presentation of vision, but one kind of excellent information carrier, which will be more important in the modern society, while computer graphics software can well promote the visual feeling and strengthen the whole presentation of advertisement design. Nowadays, national animation cannot show enough competitiveness among the work animations. Enriching the production level of animation is the significant method to acquire the differentiated development for Chinese animation, which can bring the audience more aspects through the animation design with comprehensive expressive forces. Making systemic promotion with animation scene design will be the vital means for animation products to gain the development as well as the powerful support for the future Chinese animation to spread aboard and obtain more wide development opportunities.

\section{Acknowledgements}

Subproject of the Study on the Protection and Inheritance of the Uygur Native Knowledge: "Protection and inheritance of Uygur local technology and technology" (No. 13\&ZD145).

\section{References}

[1]. Yongchun Yuan. Application of 3DMAX in the Character Animation Role Production[J]. Heilongjiang Science and Technology Information,2016(22):186.

[2]. Zhening Li, Meng Fu. Three-dimensional Simulation of Coal Mining Process Based on 3Dmax and Pro/engineer[J]. Coal Mine Modernization,2016(03):81-82+84.

[3]. Long Chen, Min Jiang. Thinking on the Application of Photoshop and 3DMAX to the Multimedia Teaching[J]. New Campus (First ten days),2015(04):81.

[4]. Qin Li. Practical Meaning and Application Thoughts of 3DMAX Applied to the Animation Production[J]. Computer Knowledge and Technology,2015,11(10):185-186+194.

[5]. Yifeng Zhang, Heping Wu, Min Sun and Huiqing Ma. Discussion on the Application of "3DMAX" Animation Production to the Curtain Wall Design with Practical Examples[J]. Doors and Windows,2012(12):1-4.

[6]. Haifeng. Application of Pro/E and 3DMAX Triode Separator Working Process[J]. Technology Information,2009(10):564+566. 